\title{
Nitrogenous Educts Through Oxidative Amidation of Phenols: The Bimolecular Reaction
}

\author{
Sylvain Canesi, Denis Bouchu and Marco A. Ciufolini*
}

Laboratoire de Synthèse et Méthodologie Organiques, CNRS UMR 5181; Université Claude Bernard Lyon 1 and Ecole Supérieure de Chimie, Physique et Electronique de Lyon, 43, Bd du 11 Novembre 1918, 69622 Villeurbanne, France, and Department of Chemistry, the University of British Columbia, 2036 Main Mall, Vancouver, BC V6T 1Z1, Canada

SUPPORTING INFORMATION 
A. Experimental protocols. Unless otherwise indicated, proton and ${ }^{13} \mathrm{C}$ NMR spectra were recorded at 300 and $75 \mathrm{MHz}$, respectively, in $\mathrm{CDCl}_{3}$ solutions. Chemical shifts are reported in ppm on the $\delta$ scale. Multiplicities are described as s (singlet), d (doublet), dd, ddd, etc. (doublet of doublets, doublet of doublets of doublets, etc.), t (triplet), q (quartet) $\mathrm{m}$ (multiplet), and further qualified as app (apparent) b (broad) c (complex). Coupling constants, $\mathrm{J}$, are reported in Hz. Low- and high-resolution mass spectra $(\mathrm{m} / \mathrm{e})$ were measured in the chemical ionization mode (CI, isobutane as the reagent gas).

B. General procedure for oxidative amidation of phenols. A solution of $\mathrm{PhI}(\mathrm{OAc})_{2}$ ("DIB", $232.0 \mathrm{mg}, 0.7 \mathrm{mmol}, 1.2$ equiv.) in $\left(\mathrm{CF}_{3}\right)_{2} \mathrm{CHOH}$ ("HFIP", $0.5 \mathrm{~mL}$ ) was added dropwise over $30 \mathrm{sec}$ to a vigorously stirred solution of a phenol ( $0.6 \mathrm{mmol}, 1$ equiv.) in $\mathrm{MeCN}(2.0 \mathrm{~mL})$ and HFIP $(1.5 \mathrm{~mL})$ kept at $15{ }^{\circ} \mathrm{C}$ (bath temperature). The mixture was stirred for $20 \mathrm{~min}$, then it was concentrated. Silica gel chromatography of the residue, first with 1:1 AcOEt / Hexanes (removal of gross contaminants), and then with $5-10 \% \mathrm{MeOH}$ in $\mathrm{CH}_{2} \mathrm{Cl}_{2}$, provided the pure product.

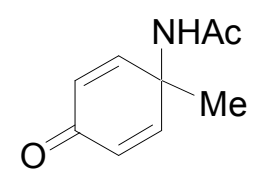

Compound 11a: Yield = 56\%. ${ }^{1} \mathbf{H}: 6.82(\mathrm{~d}, 2 \mathrm{H}, J=10.2 \mathrm{~Hz}), 6.31(\mathrm{br} \mathrm{s}, 1 \mathrm{H})$, $6.19(\mathrm{~d}, 2 \mathrm{H}, J=10.2 \mathrm{~Hz}), 1.91(\mathrm{~s}, 3 \mathrm{H}), 1.43(\mathrm{~s}, 3 \mathrm{H}) .{ }^{13} \mathrm{C}: 185.5,170.3,152.5$, 127.6, 52.5, 26.1, 23.1. HRMS: calc for $\mathrm{C}_{9} \mathrm{H}_{12} \mathrm{O}_{2} \mathrm{~N}_{1}\left(\mathrm{MH}^{+}\right)$: 166.0868 ; found:

166.0871

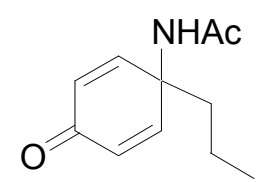

Compound 11b: Yield = 54\%. ${ }^{1} \mathbf{H}: 6.77(\mathrm{~d}, 2 \mathrm{H}, J=10.2 \mathrm{~Hz}), 6.67$ (br s, $1 \mathrm{H}), 6.24(\mathrm{~d}, 2 \mathrm{H}, J=10.2 \mathrm{~Hz}), 1.96(\mathrm{~s}, 3 \mathrm{H}), 1.69(\mathrm{~m}, 2 \mathrm{H}), 1.22(\mathrm{~m}, 2 \mathrm{H})$, 0.84 (t, 3H, $J=7.2 \mathrm{~Hz}) .{ }^{13} \mathrm{C}: 185.8,170.0,151.3,128.6,55.9,40.4,23.2$, 16.4, 13.9. HRMS: calc. for $\mathrm{C}_{11} \mathrm{H}_{16} \mathrm{O}_{2} \mathrm{~N}_{1}\left(\mathrm{MH}^{+}\right)$: 194.1181 ; found: 194.1182<smiles>CC(C)C(C)(C)NC1(C(C)C)C=CC(=O)C=C1</smiles>

Compound 11c: Yield $=62 \% .{ }^{1} \mathbf{H}: 6.81(\mathrm{~d}, 2 \mathrm{H}, J=10.2 \mathrm{~Hz}), 6.31(\mathrm{br}$, 1H), $6.26(\mathrm{~d}, 2 \mathrm{H}, J=10.2 \mathrm{~Hz}), 2.21(\mathrm{~h}, 1 \mathrm{H}, J=7.0 \mathrm{~Hz}), 1.91(\mathrm{~s}, 3 \mathrm{H}), 0,89$ (d, $6 \mathrm{H}, J=7.0 \mathrm{~Hz}) .{ }^{13} \mathrm{C}: 185.8,170.1,149.8,129.3,58.9,34.7,23.3,16.9$.

HRMS: calc for $\mathrm{C}_{11} \mathrm{H}_{16} \mathrm{O}_{2} \mathrm{~N}_{1}\left(\mathrm{MH}^{+}\right)$: 194.1181; found: 194.1181 . 


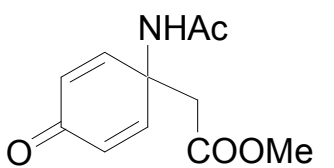

Compound 11d: Yield = 58\%. ${ }^{1} \mathbf{H}: 6.95(\mathrm{~d}, 2 \mathrm{H}, J=10.2 \mathrm{~Hz}), 6.80(\mathrm{br}$ s, 1H), $6.29(\mathrm{~d}, 2 \mathrm{H}, J=10.2 \mathrm{~Hz}), 3.72(\mathrm{~s}, 3 \mathrm{H}), 2.76(\mathrm{~s}, 2 \mathrm{H}), 1.97(\mathrm{~s}$, 3H). ${ }^{13} \mathbf{C}: 184.7,170.2,170.1,148.4,128.8,53.2,52.3,42.2,23.5$.

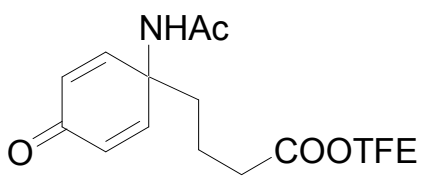

Compound 11e: Yield $=57 \% .{ }^{1} \mathbf{H}: 6.84(\mathrm{~d}, 2 \mathrm{H}, J=10.2 \mathrm{~Hz}), 6.43$ (br s, 1H), $6.28(\mathrm{~d}, 2 \mathrm{H}, J=10.2 \mathrm{~Hz}), 4.47(\mathrm{q}, 2 \mathrm{H}, J=8.3 \mathrm{~Hz})$, $2.41(\mathrm{t}, 2 \mathrm{H}, \mathrm{J}=6.8 \mathrm{~Hz}), 1.94(\mathrm{~s}, 3 \mathrm{H}), 1.83(\mathrm{~m}, 2 \mathrm{H}), 1.62(\mathrm{~m}, 2 \mathrm{H})$.

${ }^{13} \mathrm{C}: 185.4,171.4,170.0,150.0,129.2,60.5(\mathrm{q}), 55.6,37.2,32.7,23.4,18.4$.

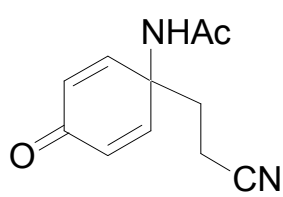

Compound 11f: Yield $=67 \% .{ }^{1} \mathbf{H}: 6.89(\mathrm{~d}, 2 \mathrm{H}, J=10.2 \mathrm{~Hz}), 6.70$ (br s, $1 \mathrm{H}), 6.31$ (d, 2H, $J=10.2 \mathrm{~Hz}), 2.26$ (br, 4H), 1.91 (s, 3H). ${ }^{13} \mathrm{C}: 184.7$, $170.5,148.0,130.0,118.7,55.0,32.4,23.4,11.9$.

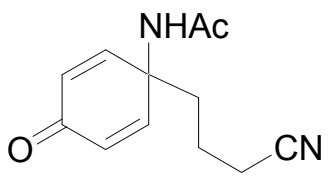

Compound 11g: Yield $=71 \% .{ }^{1} \mathbf{H}: 6.91($ br s, $1 \mathrm{H}), 6.82(\mathrm{~d}, 2 \mathrm{H}, J=$ $10.2 \mathrm{~Hz}), 6.26$ (d, 2H, $J=10.2 \mathrm{~Hz}), 2.31(\mathrm{t}, 2 \mathrm{H}, J=6.8 \mathrm{~Hz}), 1.97$ (m, 2H), 1.89 (s, 3H), 1.52 (m, 2H). ${ }^{13} \mathrm{C}: 185.3,170.2,149.9,129.1,118.8$, $55.3,36.1,23.2,19.2,16.8$.

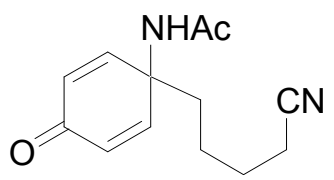

Compound 11h: Yield $=71 \% .{ }^{1} \mathbf{H}: 6.86(\mathrm{~d}, 2 \mathrm{H}, J=10.2 \mathrm{~Hz}), 6.60(\mathrm{br}$ s, 1H), $6.26(\mathrm{~d}, 2 \mathrm{H}, J=10.2 \mathrm{~Hz}), 2.31(\mathrm{t}, 2 \mathrm{H}, J=7.2 \mathrm{~Hz}), 1.92(\mathrm{~s}, 3 \mathrm{H})$, $1.87(\mathrm{~m}, 2 \mathrm{H}), 1.60(\mathrm{q}, 2 \mathrm{H}, J=7.2 \mathrm{~Hz}), 1.35(\mathrm{~m}, 2 \mathrm{H}) .{ }^{13} \mathrm{C}: 185.4,170.1$, $150.2,129.0,119.2,55.7,36.9,25.0,23.4,22.4,16.8$.

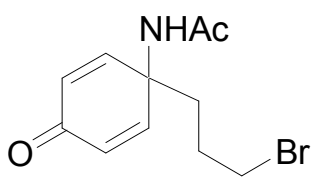

Compound 11j: Yield =65\%. ${ }^{1} \mathbf{H} 6.81(\mathrm{~d}, 2 \mathrm{H}, J=10.2 \mathrm{~Hz}), 6.37$ (br s, $1 \mathrm{H}), 6.30(\mathrm{~d}, 2 \mathrm{H}, J=10.2 \mathrm{~Hz}), 3.35(\mathrm{t}, 2 \mathrm{H}, J=6.0 \mathrm{~Hz}), 2.01(\mathrm{~m}, 2 \mathrm{H})$, $1.96(\mathrm{~s}, 3 \mathrm{H}), 1.73(\mathrm{~m}, 2 \mathrm{H}) .{ }^{13} \mathrm{C} 185.3,170.0,149.9,129.4,55.5,36.4$, $32.8,26.3,23.5$.

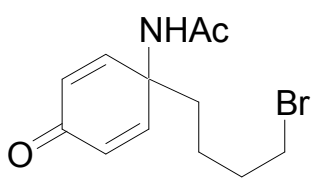

Compound 11k: Yield = 72\%. ${ }^{1} \mathbf{H}: 6.84(\mathrm{~d}, 2 \mathrm{H}, J=10.2 \mathrm{~Hz}), 6.68(\mathrm{br}$ $\mathrm{s}, 1 \mathrm{H}), 6.26(\mathrm{~d}, 2 \mathrm{H}, J=10.2 \mathrm{~Hz}), 3.32(\mathrm{t}, 2 \mathrm{H}, J=6.8 \mathrm{~Hz}), 1.91(\mathrm{~s}, 3 \mathrm{H})$, $1.80(\mathrm{~m}, 4 \mathrm{H}), 1.37(\mathrm{~m}, 2 \mathrm{H}) .{ }^{13} \mathrm{C}: 185.6,170.1,150.6,128.9,55.8,37.0$, $32.9,32.1,23.3,21.7$. 


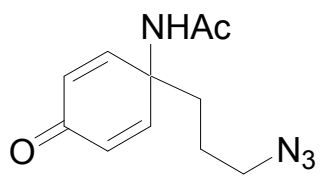

Compound 11I: Yield $=42 \% .{ }^{1} \mathbf{H}: 6.82(\mathrm{~d}, 2 \mathrm{H}, J=10.2 \mathrm{~Hz}), 6.29(\mathrm{~d}$, $2 \mathrm{H}, J=10.2 \mathrm{~Hz}$ ), 5.89. (br s, 1H), 3.31 (t, $2 \mathrm{H}, J=6.4 \mathrm{~Hz}), 1.98$ (m, 2H, overlapping a s, $3 \mathrm{H}), 1.47(\mathrm{~m}, 2 \mathrm{H}) .{ }^{13} \mathrm{C}: 185.4,170.1,150.0,129.2$, $55.6,50.9,34.9,23.4,22.9$.

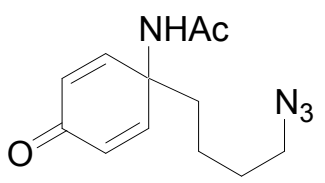

Compound 11m: Yield $=49 \%$. ${ }^{1} \mathbf{H}: 6.79(\mathrm{~d}, 2 \mathrm{H}, J=10.2 \mathrm{~Hz}), 6.25$ (br $\mathrm{s}, 1 \mathrm{H}), 6.24(\mathrm{~d}, 2 \mathrm{H}, J=10.2 \mathrm{~Hz}), 3.20$ (t, 2H, $J=7.0 \mathrm{~Hz}), 1.91(\mathrm{~s}, 3 \mathrm{H})$, $1.78(\mathrm{~m}, 2 \mathrm{H}), 1.47$ (quintuplet, $2 \mathrm{H}, J=7.0 \mathrm{~Hz}), 1.25(\mathrm{~m}, 2 \mathrm{H}) .{ }^{13} \mathrm{C}$ :

$185.6,170.2,150.7,128.8,55.8,50.8,37.4,28.4,23.2,20.4$.

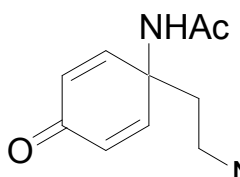

Compound 11n: Yield = 53\%. ${ }^{1} \mathbf{H}: 7.62(\mathrm{~d}, 2 \mathrm{H}, J=7.9 \mathrm{~Hz}), 7.24(\mathrm{~d}$, $2 \mathrm{H}, J=7.9 \mathrm{~Hz}), 6.98$ (br s, 1H), 6.84 (d, 2H, $J=9.8 \mathrm{~Hz}), 6.21$ (d, 2H, $J$ NHTs $=9.8 \mathrm{~Hz}), 5.83$ (br s, 1H), 2.83 (br m, 2H), 2.38 (s, 3H), 2.03 (br m, 2H), $1.91(\mathrm{~s}, 3 \mathrm{H}) .{ }^{13} \mathrm{C}: 185.6,170.8,150.4,143.8,136.1,129.8,128.9,126.9,54.8,38.2,37.7$, $23.3,21.5$.

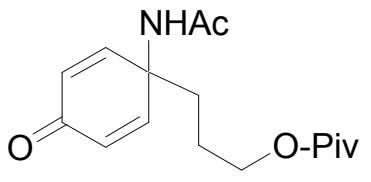

Compound 110: Yield $=67 \%$. ${ }^{1} \mathbf{H}: 6.80(\mathrm{~d}, 2 \mathrm{H}, J=10.2 \mathrm{~Hz}), 6.30$ (d, $2 \mathrm{H}, J=10.2 \mathrm{~Hz}), 6.21$ (br s, $1 \mathrm{H}), 3.99$ (t, 2H, $J=6.0 \mathrm{~Hz}), 1.95$ (s, $3 \mathrm{H}), 1.88(\mathrm{~m}, 2 \mathrm{H}), 1.53(\mathrm{~m}, 2 \mathrm{H}), 1.16(\mathrm{~s}, 9 \mathrm{H}) .{ }^{13} \mathrm{C}: 185.4,178.4$,. 169.9, 150.1, 129.1, 63.3, 55.5, 38.6, 34.2, 27.0, 23.3, 22.6. HRMS: calc. for $\mathrm{C}_{16} \mathrm{H}_{24} \mathrm{O}_{4} \mathrm{~N}_{1}$ $\left(\mathrm{MH}^{+}\right): 294.1705$; found 294.1705

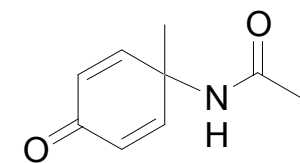

$26.2,9.3$.<smiles>CCNC1(C)C=C(Br)C(=O)C(Br)=C1</smiles>

Compound 18: Yield $=52 \% .{ }^{1} \mathbf{H}: 6.86(\mathrm{~d}, 2 \mathrm{H}, J=10.2 \mathrm{~Hz}), 6.37$ (br s, $1 \mathrm{H}), 6.21(\mathrm{~d}, 2 \mathrm{H}, J=10.2 \mathrm{~Hz}), 2.18(\mathrm{q}, 1 \mathrm{H}, J=7.5 \mathrm{~Hz}), 1.45(\mathrm{~s}, 9 \mathrm{H})$, $1.08(\mathrm{t}, 3 \mathrm{H}, J=7.5 \mathrm{~Hz}) .{ }^{13} \mathrm{C}: 185.3,173.6,152.3,127.6,52.3,29.3$, 


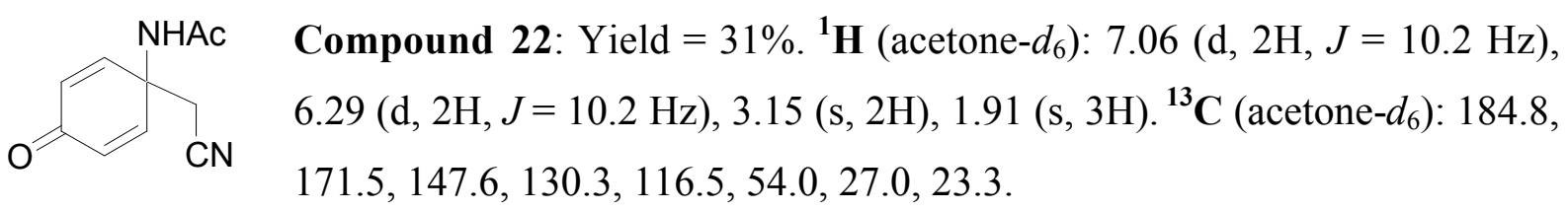

C. Procedure for the cylization of compounds $11 \mathbf{j}$ and $11 \mathbf{k}$. A solution of $11 \mathbf{j}$ or $11 \mathbf{k}(0.2$ mmol) in dry THF $(2.0 \mathrm{~mL})$ was treated with solid $\mathrm{NaH}(1.1$ eq.) and stirred at room temperature and under Ar. After 10 (11 j) / 60 (11k) minutes, TLC showed complete conversion. The mixture was quenched with 2 drops of a saturated aqueous solution of $\mathrm{NH}_{4} \mathrm{Cl}$ and concentrated, and the aqueous residue was extracted with $\mathrm{CHCl}_{3}(3 \times 2 \mathrm{~mL})$. The combined extracts were filtered and concentrated to give essentially pure product $\mathbf{2 7}$ and $\mathbf{2 8}$ as slightly yellow oils.

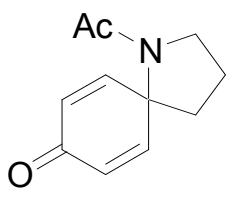

Compound 27: 1:1 ratio of amide rotamers. Yield $=91 \% .{ }^{1} \mathbf{H}: 6.89(\mathrm{~d}$, $1 \mathrm{H}, J=10.2 \mathrm{~Hz}) \& 6.76(\mathrm{~d}, 1 \mathrm{H}, J=10.2 \mathrm{~Hz}), 6.30(\mathrm{~d}, 1 \mathrm{H}, J=10.2 \mathrm{~Hz})$ \& $6.23(\mathrm{~d}, 1 \mathrm{H}, J=10.2 \mathrm{~Hz}), 3.81(\operatorname{app} \mathrm{t}, 1 \mathrm{H}, J=6.6 \mathrm{~Hz}) \& 3.72(\operatorname{app} \mathrm{t}$, $1 \mathrm{H}, J=6.6 \mathrm{~Hz}), 2.23(\operatorname{app~t}, 1 \mathrm{H}, J=6.6 \mathrm{~Hz}) \& 1.08(\mathrm{c} \mathrm{m}, 3 \mathrm{H}), 2.07(\mathrm{~s}$, $1.5 \mathrm{H}) \& 1.89(\mathrm{~s}, 1.5 \mathrm{H})$.<smiles>O=C1C=CC2(C=C1)CCCCN2C(=O)O</smiles>

Compound 28: Yield =91\%. ${ }^{1} \mathbf{H}: 7.01(\mathrm{~d}, 2 \mathrm{H}, J=10.2 \mathrm{~Hz}), 6.19(\mathrm{~d}$, $2 \mathrm{H}, J=10.2 \mathrm{~Hz}), 3.58(\operatorname{app~t}, 2 \mathrm{H}, J=5.7 \mathrm{~Hz}), 2.08(\mathrm{~s}, 3 \mathrm{H}), 1.80(\mathrm{~m}$, $2 \mathrm{H}), 1.63(\mathrm{~m}, 4 \mathrm{H}) .{ }^{13} \mathrm{C}: 185.3,171.8,152.0,125.9,56.8,38.2,23.9$, 23.2 (two overlapping signals), 19.5 . 